\title{
Preretinopic changes in the colour vision of juvenile diabetics
}

\author{
Anne Kurtenbach, Ulrich Schiefer, Andreas Neu, Eberhart Zrenner
}

\begin{abstract}
Aims-To examine the colour vision of juvenile patients suffering from diabetes mellitus without retinopathy in relation to metabolic and ophthalmic state.

Methods-Metameric matches, both Rayleigh (red/green) and Moreland (blue/ green) were used to test the colour vision yearly of 10 juvenile patients. The patients were monitored over 4 years, and during the final year, their blood glucose level was determined directly after testing colour vision. An ophthalmic examination was performed on the day of colour vision testing and blood and urine were analysed regularly throughout the 4 years. Their results are compared with an aged matched control group of 20 subjects, seven of whom were retested after 9-16 months.

Results-After 4 years, the colour vision results show an enlarged matching range for the Moreland match, as well as a smaller increase in the matching range for the Rayleigh match. No significant correlation was found between blood glucose at the time of testing and any of the variables measured.

Conclusion-The pattern of colour vision deficits in metameric matching shown by juvenile diabetics is consistent with postreceptoral alterations of the inner retina, at this preretinopic stage of disease. Duration of diabetes is correlated with both colour vision changes and morphological alteration of the retina.

(Br f Ophthalmol 1999;83:43-46)
\end{abstract}

University Eye

Hospital, Department of Pathology of Vision and Neuro-

ophthalmology,

Tübingen, Germany

A Kurtenbach

U Schiefer

E Zrenner

University Children's Hospital, Tübingen, Germany

A Neu

Correspondence to: Anne Kurtenbach, University Eye Hospital, Department of Pathology of Vision and Neuroophthalmology,

Schleichstrasse 12-16, 72076

Tübingen, Germany.

Accepted for publication 31 July 1998
It is well documented that patients suffering from diabetes mellitus (type 1) can show alterations in their colour perception. Metabolic alterations caused by chronically raised blood glucose levels cause capillary degeneration, hypoxia, and cell death in the retina of diabetics. The role of hypoxia in the colour vision changes has recently been stressed by the findings of Dean et al, ${ }^{1}$ who show that the colour deficits in diabetics with retinopathy can be partially reversed by inhalation of oxygen. Nevertheless, the aetiology of diabetic retinopathy remains unclear, and the initial retinal sites affected by the hypoxia are as yet unknown. Most studies have been performed on patients with diabetic retinopathy and report a deficiency at the short end of the spectrum. Alterations have been found in photoreceptor function ${ }^{2-5}$ as well at the postreceptoral stage of retinal processing, ${ }^{4-7}$ with some patients showing deficits at both retinal loci.
The aim of this study was to obtain more information about the initial alterations in the diabetic eye. In a prospective study, we monitored 10 juvenile patients (aged 12-18 years) without retinopathy over a period of 4 years to gain information about the early changes in their colour vision. To test the colour vision we used metameric matchesthat is, equating a mixture of two monochromatic colours with a third comparison wavelength. The most common metameric match is based on the Rayleigh equation, where green and red monochromatic test lights are mixed to match a third, yellow. The proportion of mixed lights used to obtain a match is given as the match midpoint (MMP), the range of mixtures which will be accepted as matching the reference yellow is given as the matching range (MR). As well as the Rayleigh match, we also examined the Moreland match which, with mixtures of blue and green, test the sensitivity of the short wavelength pathway.

In this study, we examined the type and size of errors made with metameric matches to help identify the site or sites affected at this preretinopic stage of the disease. We also compared these variables with metabolic control and ophthalmic state over the period tested. Although it is generally accepted that any perceptual deficit is due to a raised blood glucose level, significant correlations are often difficult to ascertain. In the final year of testing, we also examined the blood glucose level at the time of colour vision testing, which has been shown to affect the sensitivity of the short wavelength sensitive pathway. ${ }^{8}$ The results of the diabetic group are compared with those of an aged matched control group. The colour vision results found for these patients after 3 years have been reported. ${ }^{9}$

\section{Materials and methods}

SUBJECTS

Ten insulin dependent diabetics (four male, six female) participated in the tests which were performed once a year over a 4 year period (eight patients in years 1 and 2, 10 in years 3 and 4). Six patients were common to all years. They were aged between 12 and 18 years (mean 15.9 (SD 2.0)) in the first year of testing and had suffered diabetes for between 3.6 and 14.7 years (mean 9.6 (3.8)). Nine patients showed no retinopathy and one patient very mild retinopathy (six microaneurysms), as evidenced from ophthalmoscopy and colour fundus photographs. Visual acuity and intraocular pressure were normal. The patients had attended the Children's Hospital, Tübingen on average every 8-10 weeks since detection of the 
disease, where blood was analysed. In the final year capillary blood glucose levels were determined directly after testing for nine of the patients. Lipid metabolism (cholesterin and triglyceride) were also monitored about two to three times per year, as well as kidney function (creatinine, urea and/or $\beta 2$ microalbumin).

The colour vision results of the diabetic group are compared with an age matched control group of 20 non-diabetic subjects (11 male, nine female), aged between 12 and 24 years (mean 19.4 (3.6)). They were included in the study if they showed normal colour vision on the Lanthony D-15 or Farnsworth-Munsell 100 hue tests and had normal visual acuity. Seven were retested after 9-16 months.

\section{APPARATUS}

We determined Rayleigh and Moreland matches in one eye of each patient, using a computer controlled electronic anomaloscope (Interzeag, Schlieren, Switzerland). For the Rayleigh equation, green $(545 \mathrm{~nm})$ and red $(670 \mathrm{~nm})$ primaries are mixed to produce a yellow (589 $\mathrm{nm}$ ), whereas for the Moreland equation blue $(436 \mathrm{~nm})$ and green $(490 \mathrm{~nm})$ primaries are mixed to produce a desaturated blue green ( 480 $\mathrm{nm}$ ). The primaries in the four independent channels are produced using interference filters (10 nm bandwidth). The stimulus is a circular bipartite field of 2 degree diameter and is presented to one eye for 2 seconds, with an achromatic field between presentations to maintain the adaptation level. The ratio of the two primaries is varied in a standardised procedure. The computer controls all settings and presents, pseudorandomly, a set of predefined mixtures of the primaries.
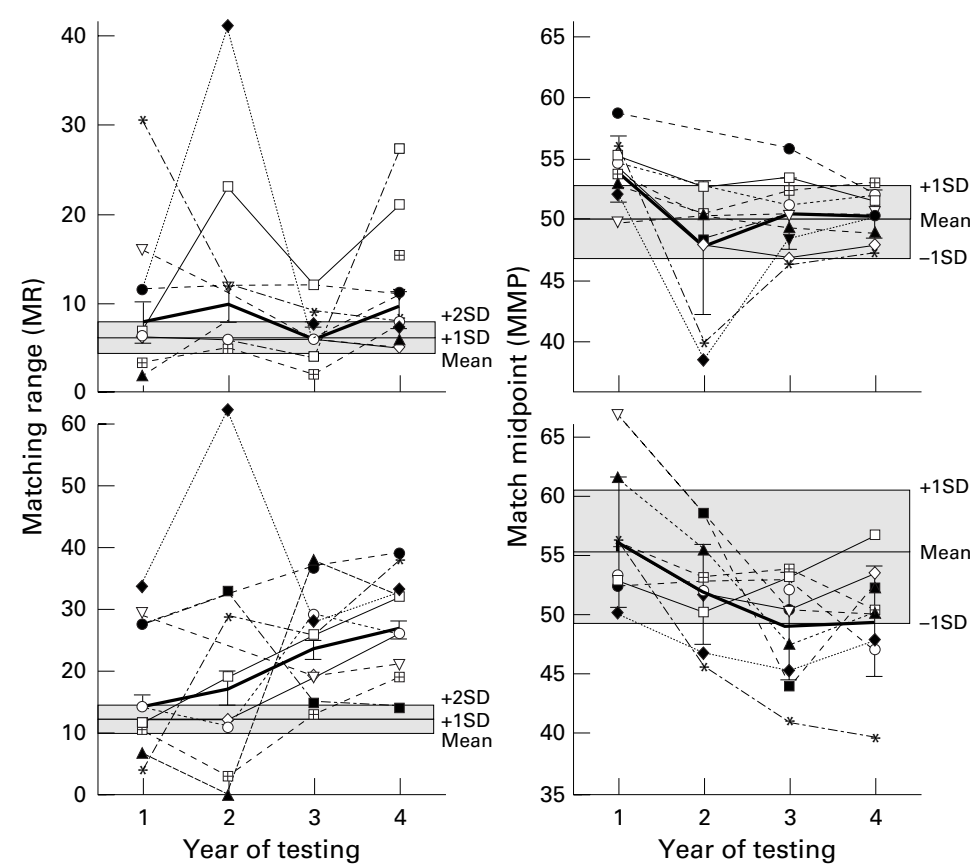

Figure 1 Individual results of Rayleigh (upper panels) and Moreland matches (lower panels) obtained from 10 juvenile diabetics tested once a year over 4 years ( $X$ axis). The match range (MR) is shown on the left, the match midpoint (MMP) on the right. Individual subjects are shown by different symbols. The thick line indicates the geometric mean for the match range and the mean of the match midpoint. Also shown by the shaded area are the average results of the control group $+1 S D$ and $+2 S D$ for the MRs and \pm 1 $S D$ for the MMPs.
PROCEDURE

The subjects' colour vision was first tested. This was followed by the ophthalmological examination, which included visual acuity, slit lamp ophthalmoscopy, intraocular pressure, fundus (indirect and direct ophthalmoscopy), and fundus photography. Fundus examination was performed with a dilated pupil (tropicamide, Mydriaticum Stulln (Pharma Stulln $\mathrm{GmbH})$. Linear regressions were fitted to the data using STATVIEW for the Macintosh.

\section{Results}

Ophthalmic examination revealed no lens or intraocular pressure changes over the period tested. Fundus photography showed that two patients exhibited a sporadic appearance and disappearance of isolated microaneurysms. These two patients had the longest duration of disease in the group (14.7 and 13.3 years at year one of testing).

The results from the colour vision testing can be seen in Figure 1. We show the patients' individual results by different symbols, from all 4 years tested (X axis) for both Rayleigh (upper graphs) and Moreland (lower graphs) matches. The thick line shows the average results of the group: The match range (MR) shown on the left, which is not normally distributed, is given as the geometric mean (1 SD); the mean match midpoint (MMP) (1 SD) is shown on the right, depicted on a scale of maximal 100 units where 0 indicates only the green primary for the Rayleigh equation and only the blue primary for the Moreland equation. Also shown in Figure 1 by the shaded area, are the results of the control group for year one. For the MR we show the geometric mean $+1 \mathrm{SD}$ and $+2 \mathrm{SD}$; for the MMP we show the mean results (plus or minus $1 \mathrm{SD}$ ). The control group values are very similar in the second year of testing (Rayleigh: MR: 5.51 (1.74), MMP: 49.57 (3.21); Moreland: MMP: 55.06 (5.86), MR: $12.02(1.09))$.

The mean Rayleigh MR (upper left) for the diabetic group varies around the mean +2 SD values of the control group. Many patients give significantly widened ranges, results being considered significant if they are larger than the 95 th percentile (geometric mean $+2 \mathrm{SD}$ ) of the control group. In the last year of testing four of the 10 patients showed a significantly increased Rayleigh MR. The Moreland MR (lower left) shows a continual widening over the years. In the last year of testing, nine of the 10 patients gave significant Moreland MR results The two patients who developed sporadic microaneurysms gave a raised score for both matches. The individual results for both Rayleigh and Moreland match midpoints (right), on the other hand, seldom show results significantly different from those of the control group. One subject shows a significant decrease in the Moreland match midpoint. As a group therefore, the diabetics show a progressively worsening colour vision deficit, manifest at this stage of the disease by a significantly increased match range for the Moreland equation.

The match range values we obtained for our control group of juveniles are slightly higher 

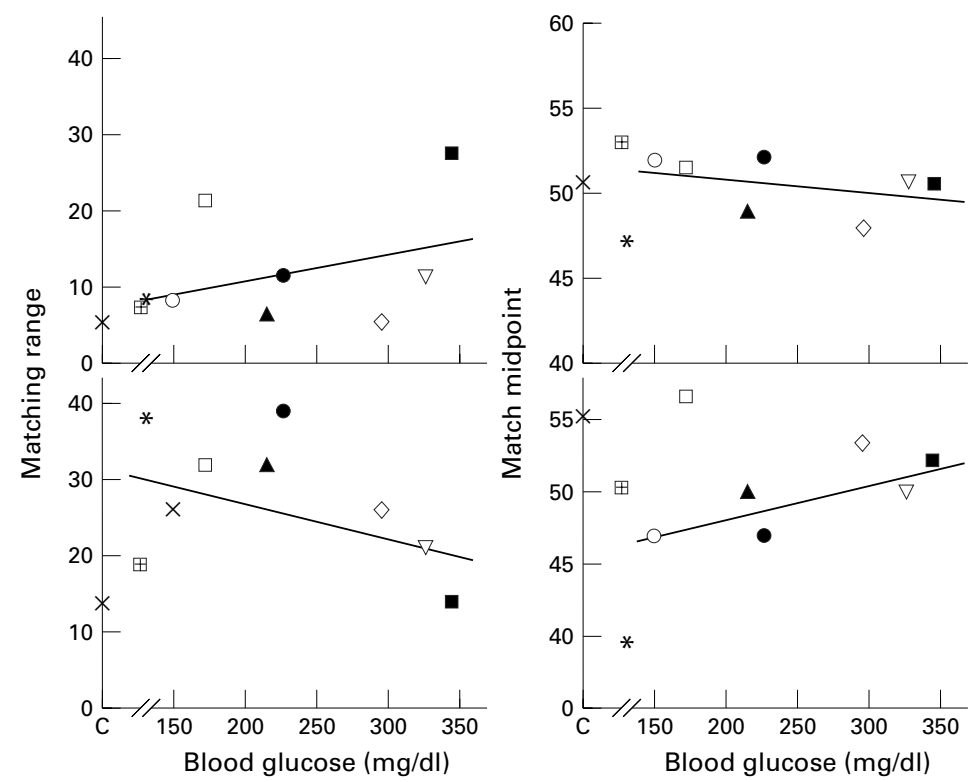

Figure 2 Capillary blood glucose ( $X$ axis) in comparison with the results of metameric matching in the final year of testing. The upper two panels show the results of the Rayleigh match, the lower panels the results for the Moreland match. Match ranges (MR) are shown to the left, match midpoints to the right. At $C$ the mean results of the control group are shown ( $x$ ). Regression lines have been fitted to the data points (Rayleigh $M R ; R=0.19$, $p=0.64:$ Moreland MR; $R=0.41, p=0.26:$ Rayleigh $M M P ; R=0.30, p=0.41:$ Moreland $M M P ; R=0.44, p=0.23)$. The symbol used for each subject is the same as in Figure 1.
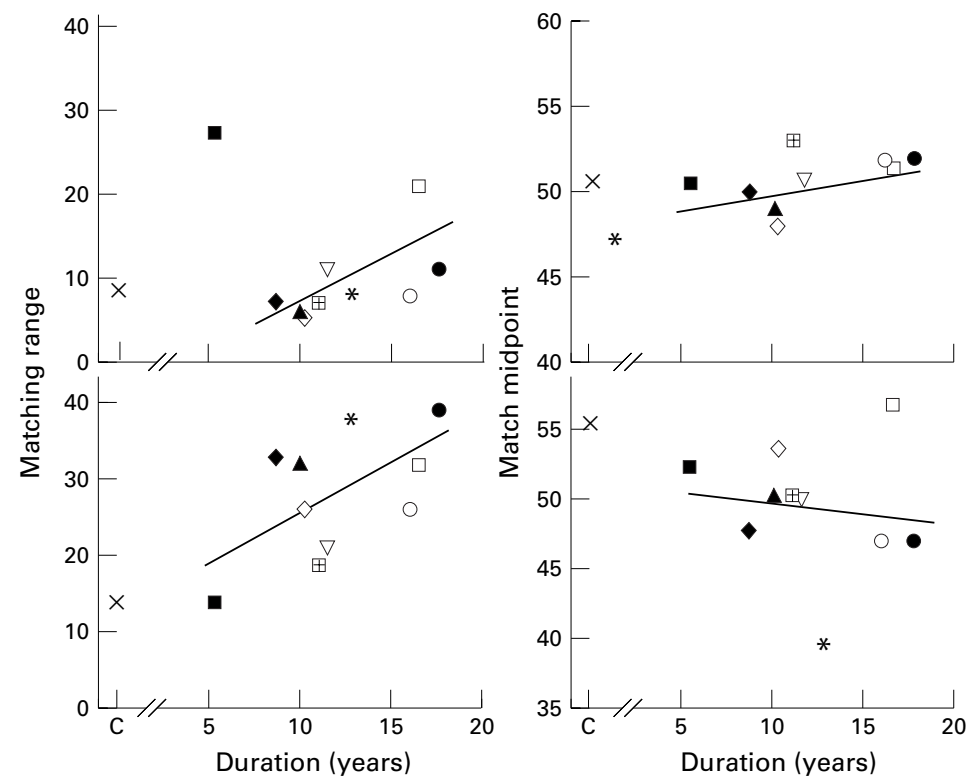

Figure 3 As for Figure 2 with duration of disease ( $X$ axis) in comparison with the results of metameric matching. The coefficients for the linear regressions are: Rayleigh $M R$; $R=0.59, p=0.05:$ Moreland $M R ; R=0.62, p=0.05:$ Rayleigh $M M P ; R=0.23, p=0.48$ : Moreland $M M P ; R=0.62, p=0.48$.

than those provided as a guide by the manufacturer to the normal population based on around 50 subjects (personal communication). The 95th percentile of the Rayleigh MR is given as 4, that of the Moreland MR as 12 . For the Moreland match, the mean population MMP for the Rayleigh match is given as 50 (3.5), that for the Moreland match 53 (5.0).

Blood glucose levels $\left(\mathrm{gHb}\right.$ and $\mathrm{HbA}_{1 \mathrm{c}}$ ) were ascertained within 6 weeks of testing. The median varied around the upper limits of those aimed at in diabetes therapy $\left(<7.5 \%\right.$ for $\mathrm{HbA}_{1 \mathrm{c}}$ and $<10 \%$ for $\mathrm{gHb}$ ), but there was a large variation between subjects. The average yearly values for urea and creatinine remained within population recommended values, although the latter showed a small steady increase over the years. The values for triglyceride and cholesterin varied around the upper population limit.

To examine more closely the relation between blood glucose and colour vision results, we compared for each subject the blood glucose $(\mathrm{gHb})$ and the results of the Rayleigh and Moreland anomaloscope matches. Consideration of each year separately yielded no significant correlations, although there was a tendency for higher $\mathrm{gHb}$ and $\mathrm{HbA}_{1 \mathrm{c}}$ values to result in a Rayleigh MMP that was shifted to green.

These variable results led us to measure capillary blood glucose directly after testing colour vision during the final year. The results for nine subjects can be seen in the scatter plot in Figure 2. On the $\mathrm{X}$ axis we show the measured glucose level, on the $\mathrm{Y}$ axis the MR (left) and MMP (right) of the Rayleigh (upper graphs) and Moreland (lower graphs) matches. On the $\mathrm{Y}$ axis (C), we also show the mean values obtained for these variables from the control group (x). Regression lines have been fitted to the results; there are no significant correlations.

In Figure 3 we show for comparison the same results, but plotted as a function of duration of disease. There is a significant correlation $(p<0.05)$ between duration of diabetes and the Moreland MR, and, omitting one extreme value, for nine of the 10 patients tested, also with the Rayleigh MR.

\section{Discussion}

In this study we examined the changes in colour vision over 4 years in juvenile diabetic patients, two of whom showed some visible change in their retinal vascular state. We also monitored metabolic control and studied the effect of capillary blood glucose levels measured at the time of testing on the colour vision results.

\section{COLOUR VISION CHANGES}

Our diabetics are associated with two main colour deficits. The most prominent is a widening of the mean matching range of the Moreland match that is present in the first year of testing and which becomes significantly larger with time (Fig 1). In the fourth year of testing, nine of the 10 patients showed significant changes, and there is a significant relation between duration of disease and this enlargement (Fig 3). Secondly, some of the patients show a widening of the Rayleigh MR, also associated with the duration of the disease (Fig 3 ). An enlarged match range indicates that the colour discrimination ability is worsening in these patients. Such a task relies in general on neuronal interactions of the inner retina, where photoreceptor signals are compared, although a relatively extreme loss of a large number of photoreceptors, unlikely in these otherwise healthy juveniles, could also cause such a deficit. Therefore, initial changes in the diabetic eye are occurring at the postreceptoral level, especially involving the opponency of the short wavelength sensitive cone. The results support 
those of Kinnear et $a l,{ }^{10}$ who found a deficit in blue discriminability which increased with age, using the Pickford-Nicolson anomaloscope.

On the whole, alterations of preretinal structures or of photoreceptors, will be evidenced by changes in the match midpoints; the ratio of the mixture of the two primaries. Lens changes are not seen in the ophthalmic examination of our patients, although small changes not detectable by slit lamp ophthalmoscopy cannot be ruled out. We find no change in the Rayleigh match midpoint, but a small but persistent shift towards blue in the match midpoint of the Moreland match in the average results of the diabetic group. Eight of the 10 patients gave lower values in year 4 of testing compared with year 1. This difference is not significant in these juveniles, but in a group of adult diabetics with retinopathy, such a pseudotritanopia is found, as well as to a lesser extent, a widening of the Moreland MR. ${ }^{11}$ It may be that the short wavelength sensitive photoreceptor is only weakly affected at this early stage of the disease in juveniles. We cannot, however, rule out a reduction in the absolute sensitivity of one or more cone pathways in our diabetics, caused, for example, by a reduction in photoreceptor numbers, which would not be detected with this method.

\section{RELATION BETWEEN THE COLOUR VISION}

CHANGES AND METABOLIC CONTROL

As a group, the metabolic control of the juvenile diabetics remained relatively stable over the test period. Small increases in creatinine were found, which is normal for patients of this age group. Values for $\mathrm{gHb}$ and $\mathrm{HbA}_{1 \mathrm{c}}$ also rise during puberty. For each patient, however, there were sometimes large variations in the blood glucose over this time.

None of the metabolic variables measured show significant correlations to the values obtained in the colour vision tests. We also find that the capillary blood glucose levels measured directly after testing has no consistent effect on the results of metameric matching (Fig 2), although a larger sample of patients is required to verify this. A decrease in the sensi- tivity of the short wavelength pathway ${ }^{8}$ has been shown during acute elevation of blood glucose in diabetics. ${ }^{8}$ Our results agree with those of North et $a l,{ }^{12}$ who also find no evidence for colour vision changes in hyperglycaemic patients.

Using the method of metameric matching we find that the most prominent initial change in the preretinopic eye of diabetic patients can be explained by changes occurring postreceptorally at the inner layer of the retina. Duration of disease is the most important factor in our group of diabetic subjects for the development of colour vision and morphological changes (Fig 3), as reported in previous studies. ${ }^{10}$ The two patients who have had diabetes the longest, show the first signs of a retinopathy, and a deterioration in both blue-green and red-green discrimination.

We thank Dr E Apfelstedt, Dr L Rüttiger, Dr H Knau, and C Fach for their help in collecting some of the data. Part of this work was supported by the Wilhelm Sander-Stiftung, Munich.

1 Dean F, Dornhurst A, Arden GB. Partial reversal of protan and tritan colour defects with inhaled oxygen in insulin dependent subjects. Br f Ophthalmol 1997;81:27-30.

2 Elsner AE, Burns SA, Lobes LA, et al. Cone photopigment bleaching abnormalities in diabetes. Invest Ophthalmol Vis Sci 1987;28:718-24.

3 Yamamoto S, Kamiyama, M, Nitta K, et al. Selective reduction of the $\mathrm{S}$ cone electroretinogram in diabetes. $\mathrm{Br} \mathcal{F} \mathrm{Oph}$ thalmol 1996;80:973-5.

4 Holopigian K, Greenstein VC, Seile W, et al. Evidence for photoreceptor changes in patients with diabetic retino-
pathy. Invest Ophthalmol Vis Sci 1997;39:2355-65.

pathy. Invest Ophthalmol Vis Sci 1997;39:2355-65.
5 Bresnick GH, Palta M. Temporal aspects of the elecBresnick GH, Palta M. Temporal aspects of the elec-
troretinogram in diabetic retinopathy. Arch Ophthalmol troretinogram in

6 Schefrin BE, Adams AJ, Werner JS. Anomalies beyond sites of chromatic opponency contribute to sensitivity losses of an S-cone pathway in diabetes. Clin Vis Sci 1991;6:219-28.

7 Greenstein VC, Shapiro A, Zaidi Q, et al. Psychophysical evidence for post-receptoral sensitivity loss in diabetics. Invest Ophthalmol Vis Sci 1992;33:2781-90.

8 Volbrecht VJ, Schneck ME, Adams AJ, et al. Diabetic shortwavelength sensitivity: variations with induced changes in
blood glucose level. Invest Ophthalmol Vis Sci 1994;35: 1243-6.

9 Kurtenbach A, Schiefer U, Zrenner E, et al. Juvenile diabetics and the color vision meter. In: Cavonius CR, ed. Colour ics and the color vision meter. In: Cavonius CR, ed. Colour
vision deficiencies XIII. Dordrecht: Kluwer, 1997:205-10.

10 Kinnear PR, Aspinall PA, Lakowski R. The diabetic eye and colour vision. Trans Ophthalmol Soc UK 1972;92:69-78.

11 Hermès D, Roth A, Borot N. The two equation method. II. Results in retinal and optic nerve disorders. In: Drum B, Verriest G, eds. Colour vision deficiencies X. Dordrecht Kluwer, 1989:205-10.

12 North RV, Cooney O, Chambers D, et al. Does hyperglycaemia have an influence upon colour vision of patients with diabetes mellitus? Ophthal Physiol Opt 1997;17:95-101. 